\title{
Search for solar Kaluza-Klein axions in theories of low-scale quantum gravity
}

\author{
L. Di Lella, ${ }^{1}$ A. Pilaftsis, ${ }^{2}$ G. Raffelt,,${ }^{3,4}$ and K. Zioutas ${ }^{1,5}$ \\ ${ }^{1}$ CERN, CH-1211 Geneva 23, Switzerland \\ ${ }^{2}$ Institut für Theoretische Physik, Universität Würzburg, Am Hubland, 97074 Würzburg, Germany \\ ${ }^{3}$ Max-Planck-Institut für Physik, Föhringer Ring 6, 80805 Munich, Germany \\ ${ }^{4}$ TECHNION (Israel Institute of Technology), Haifa 32000, Israel \\ ${ }^{5}$ Department of Physics, University of Thessaloniki, GR 54006 Thessaloniki, Greece
}

(Received 5 July 2000; published 22 November 2000)

\begin{abstract}
We explore the physics potential of a terrestrial detector for observing axionic Kaluza-Klein excitations coming from the Sun within the context of higher-dimensional theories of low-scale quantum gravity. In these theories, the heavier Kaluza-Klein axions are relatively short-lived and may be detected by a coincidental triggering of their two-photon decay mode. Because of the expected high multiplicity of the solar axionic excitations, we find experimental sensitivity to a fundamental Peccei-Quinn axion mass up to $10^{-2} \mathrm{eV}$ (corresponding to an effective axion-photon coupling $g_{a \gamma \gamma} \approx 2.0 \times 10^{-12} \mathrm{GeV}^{-1}$ ) in theories with 2 extra dimensions and a fundamental quantum-gravity scale $M_{\mathrm{F}}$ of order $100 \mathrm{TeV}$, and up to $3.0 \times 10^{-3} \mathrm{eV}$ (corresponding to $g_{a \gamma \gamma} \approx 6.0 \times 10^{-13} \mathrm{GeV}^{-1}$ ) in theories with 3 extra dimensions and $M_{\mathrm{F}}=1 \mathrm{TeV}$. For comparison, based on recent data obtained from lowest level underground experiments, we derive the experimental limits $g_{a \gamma \gamma}$ $\lessgtr 2.5 \times 10^{-11} \mathrm{GeV}^{-1}$ and $g_{a \gamma \gamma} \lesssim 1.2 \times 10^{-11} \mathrm{GeV}^{-1}$ in the aforementioned theories with 2 and 3 large compact dimensions, respectively.
\end{abstract}

PACS number(s): $14.80 . \mathrm{Mz}$

\section{INTRODUCTION}

In superstring theories it turns out to be possible to lower the string scale without lowering the Planck scale [1-6]. Most notably, Arkani-Hamed, Dimopoulos and Dvali [5] have proposed the radical possibility that the fundamental scale of quantum gravity might no longer be associated with the Planck mass $M_{\mathrm{P}}=1.2 \times 10^{19} \mathrm{GeV}$, but the true scale of quantum gravity, $M_{\mathrm{F}}$, could be many orders of magnitude smaller than $M_{\mathrm{P}}$, close to $\mathrm{TeV}$ energies. In such a novel theoretical framework, the standard-model (SM) particles can only live in a (1+3)-dimensional Minkowski subspace that constitutes our observable world, whereas gravity may freely propagate to a number $n$ of large extra dimensions. Furthermore, the ordinary Planck mass $M_{\mathrm{P}}$ would be related to the genuinely fundamental scale $M_{\mathrm{F}}$ through

$$
M_{\mathrm{P}} \approx M_{\mathrm{F}}\left(R M_{\mathrm{F}}\right)^{n / 2},
$$

where $R$ denotes the compactification radius, which is considered to be common for all extra compact dimensions. The case $n=1$ and $M_{\mathrm{F}}$ of order $\mathrm{TeV}$ leads to a visible macroscopic compactification radius and is therefore not viable. Moreover, astrophysical and cosmological considerations give rise to a lower limit on $M_{\mathrm{F}}$ of order $100 \mathrm{TeV}$, for the scenario with $n=2$ extra dimensions [7], while $M_{\mathrm{F}}$ can be as low as $1 \mathrm{TeV}$ for theories with $n>2$ dimensions.

In addition to gravity, one might think that fields which are singlets under the standard-model gauge group could also propagate in the $[1+(3+n)]$-dimensional space. As such, one might consider isosinglet neutrinos [8-10] or axion fields $[5,11,12]$. In fact, within the context of theories of $\mathrm{TeV}$-scale quantum gravity, the latter realization is theoretically compelling for the solution of the strong $C P$ problem through the Peccei-Quinn (PQ) mechanism. According to this idea, the strong $C P$-odd parameter $\theta$ may be dynamically eliminated by means of the spontaneous breakdown of a global U(1) symmetry. On the other hand, phenomenological and astrophysical considerations place lower and upper limits on the breaking scale $v_{\mathrm{PQ}}$ of the PQ-U(1) symmetry, which has to be many orders of magnitude larger than the $\mathrm{TeV}$ scale of quantum gravity. Therefore, in order to account for this large mass scale, one inevitably has to introduce a singlet higher-dimensional axion field into the QCD Lagrangian, with a higher-dimensional PQ-breaking scale $\bar{v}_{\mathrm{PQ}}$ that could even be much smaller than $1 \mathrm{TeV}$. As we will see below, as a result of the compactification of the large extra dimensions, the effective four-dimensional PQ-breaking scale $v_{\mathrm{PQ}}$ can be obtained from $\bar{v}_{\mathrm{PQ}}$, after multiplying the latter by the huge higher-dimensional volume factor $\left(M_{\mathrm{F}} R\right)^{n / 2} \approx M_{\mathrm{P}} / M_{\mathrm{F}}$. In this way, the PQ-breaking scale $v_{\mathrm{PQ}}$ may reside in the phenomenologically allowed region. Another feature of the higher-dimensional axionic theories is that their mass spectrum consists of a tower of Kaluza-Klein (KK) excitations, which have an almost equidistant mass spacing of order $1 / R$. The lowest KK excitation may be identified with the ordinary PQ axion and specifies the strength of each KK state to matter.

This tower of axionic modes has two phenomenological consequences. First, for a fixed value of the axion coupling constant to matter or photons, a given source such as the Sun will emit axions of each mode up to the kinematic limit. The high multiplicity of the KK axion modes thus leads to much larger flux than would be otherwise expected. Second, the large mass of the KK modes compared to usual axions dramatically increases the width of the decay process $a \rightarrow \gamma \gamma$ by opening up phase space. Therefore, one may plausibly search for the decay photons of the solar KK axion flux in a laboratory experiment. 
In this paper, we shall analyze the potential of a terrestrial axion detector to observe the radiative decay of solar KK axion modes. In particular, such a detector proves inexpensive and may run in parallel with the CERN Axion Solar Telescope (CAST) which will be built from a decommissioned LHC test magnet [13]. We will find that the suggested terrestrial detector may reach the unprecedented sensitivity of the $10^{-2}-\mathrm{eV}$ level $\left(g_{a \gamma \gamma} \approx 10^{-12} \mathrm{GeV}^{-1}\right)$ to the fundamental PQ axion mass $\left(m_{\mathrm{PQ}}\right)$.

The paper is organized as follows: in Sec. II we briefly describe the basic low-energy structure of a generic theory that includes higher-dimensional axions. In Sec. III we compute the solar flux of massive KK axions. In Sec. IV we estimate the event rates of photons due to axion decays as seen by a terrestrial detector. Section V summarizes our conclusions.

\section{AXIONS IN LARGE EXTRA DIMENSIONS}

Before discussing the higher-dimensional case, let us first recall the main phenomenological predictions of the axion theories in four dimensions. The axionic sector of the effective Lagrangian which is of interest to us has the generic form

$$
\mathcal{L}_{\text {eff }}=\frac{1}{2}\left(\partial_{\mu} a\right)\left(\partial^{\mu} a\right)-\frac{1}{2} m_{\mathrm{PQ}}^{2} a^{2}+\frac{g_{a \gamma \gamma}}{4} a F_{\mu \nu} \widetilde{F}^{\mu \nu},
$$

where $a$ is the PQ axion, $F_{\mu \nu}$ and $\widetilde{F}^{\mu \nu}$ are the electromagnetic field-strength tensor and its associate dual tensor, and

$$
g_{a \gamma \gamma}=\frac{\xi \alpha_{\mathrm{em}}}{\pi} \frac{1}{v_{\mathrm{PQ}}}
$$

is the effective axion-photon-photon coupling. The multiplicative parameter $\xi$ in Eq. (2.2) is generally of order unity, and crucially depends on the axion model under study $[14,15]$. Furthermore, the PQ axion mass $m_{\mathrm{PQ}}$ is related to the breaking scale $v_{\mathrm{PQ}}$ of the PQ U(1) symmetry through

$$
m_{\mathrm{PQ}} \sim \frac{m_{\pi}^{2}}{v_{\mathrm{PQ}}},
$$

where $m_{\pi} \approx 135 \mathrm{MeV}$ is the pion mass. Astrophysical and cosmological limits [16] indicate that

$$
10^{9} \mathrm{GeV} \lesssim v_{\mathrm{PQ}} \lesssim 10^{12} \mathrm{GeV},
$$

which in turn by virtue of Eq. (2.3) implies that

$$
10^{-2} \mathrm{eV} \gtrsim m_{\mathrm{PQ}} \gtrsim 10^{-5} \mathrm{eV},
$$

respectively. The lifetime of the PQ axion is easily calculated to be

$$
\begin{aligned}
\tau(a \rightarrow \gamma \gamma)= & \frac{64 \pi}{g_{a \gamma \gamma}^{2} m_{\mathrm{PQ}}^{3}} \approx 10^{48} \text { days } \\
& \times\left(\frac{10^{-15} \mathrm{GeV}^{-1}}{g_{a \gamma \gamma}}\right)^{2}\left(\frac{10^{-5} \mathrm{eV}}{m_{\mathrm{PQ}}}\right)^{3} .
\end{aligned}
$$

For $g_{a \gamma \gamma}=10^{-15} \mathrm{GeV}^{-1}$, which corresponds to $m_{\mathrm{PQ}}$ $=10^{-5} \mathrm{eV}$, the axion lifetime turns out to be much larger than the age of the universe. The prospect of detecting photonic axion decays would have remained hopeless, even if one had considered larger axion masses. For instance, for $m_{\mathrm{PQ}}=10^{-1} \mathrm{eV}\left(g_{a \gamma \gamma}=10^{-11} \mathrm{GeV}^{-1}\right)$, the axion decay is still undetectable with a lifetime $\tau(a \rightarrow \gamma \gamma) \approx 10^{27}$ days.

We shall now focus on the higher-dimensional case. Following Refs. [5,11,12], we introduce one singlet axion field $a\left(x^{\mu}, \mathbf{y}\right)$ which feels the presence of a number $\delta \leqslant n$ of large extra dimensions, denoted by $\mathbf{y}=\left(y_{1}, y_{2}, \ldots, y_{\delta}\right)$. The relevant axionic sector may then be determined by the effective Lagrangian

$$
\begin{aligned}
\mathcal{L}_{\mathrm{eff}}= & \int d^{\delta} \mathbf{y}\left[\frac{1}{2} M_{\mathrm{F}}^{\delta}\left(\partial_{\mu} a\right)\left(\partial^{\mu} a\right)+\frac{1}{2} M_{\mathrm{F}}^{\delta}\left(\partial_{\delta} a\right)\left(\partial^{\delta} a\right)\right. \\
& \left.+\delta^{(\delta)}(\mathbf{y}) \frac{\xi \alpha_{\mathrm{em}}}{\pi} \frac{a}{\bar{v}_{\mathrm{PQ}}} F_{\mu \nu} \widetilde{F}^{\mu \nu}\right]
\end{aligned}
$$

where $\bar{v}_{\mathrm{PQ}}$ denotes the original higher-dimensional PQbreaking scale. In Eq. (2.7), the axion field is compactified on a $\mathbf{Z}_{2}$ orbifold with an orbifold action [12]: $\mathbf{y} \rightarrow-\mathbf{y}$, i.e., the axion field satisfies the properties $a\left(x^{\mu}, \mathbf{y}\right)=a\left(x^{\mu}, \mathbf{y}+2 \pi R\right)$ and $a\left(x^{\mu}, \mathbf{y}\right)=a\left(x^{\mu},-\mathbf{y}\right)$. The latter gives rise to the $\mathrm{KK}$ decomposition:

$$
a\left(x^{\mu}, \mathbf{y}\right)=\sum_{\mathbf{n}=\mathbf{0}}^{\infty} a_{\mathbf{n}}\left(x^{\mu}\right) \cos \left(\frac{\mathbf{n y}}{R}\right),
$$

where $\mathbf{n}=\left(n_{1}, n_{2}, \ldots, n_{\delta}\right)$ is a $\delta$-dimensional vector that labels the individual KK excitations, and $\sum_{\mathbf{n}=\mathbf{0}}^{\infty} \equiv \sum_{n_{1}=0}^{\infty} \sum_{n_{2}=0}^{\infty}$ $\cdots \sum_{n_{\delta}=0}^{\infty}$. Substituting Eq. (2.8) into Eq. (2.7) and taking the PQ mechanism into consideration, we arrive at the effective Lagrangian [12]

$$
\begin{aligned}
\mathcal{L}_{\mathrm{eff}}= & \frac{1}{2} \sum_{\mathbf{n}=\mathbf{0}}^{\infty}\left(\partial_{\mu} a_{\mathbf{n}}\right)\left(\partial^{\mu} a_{\mathbf{n}}\right)-\frac{1}{2} m_{\mathrm{PQ}}^{2} a_{0}^{2} \\
& -\frac{1}{2} \sum_{\mathbf{n} \neq \mathbf{0}}^{\infty} \frac{\mathbf{n}^{2}}{R^{2}} a_{\mathbf{n}}^{2}+\frac{\xi \alpha_{\mathrm{em}}}{\pi} \sum_{\mathbf{n}=\mathbf{0}}^{\infty} \frac{r_{\mathbf{n}} a_{\mathbf{n}}}{v_{\mathrm{PQ}}} F_{\mu \nu} \widetilde{F}^{\mu \nu},
\end{aligned}
$$

with $r_{0}=1$ and $r_{\mathbf{n} \neq \mathbf{0}}=\sqrt{2}$. From Eq. (2.9), it is easy to read off the effective couplings of the KK axions to photons,

$$
g_{a_{\mathbf{n}} \gamma \gamma}=\frac{r_{\mathbf{n}} \xi \alpha_{\mathrm{em}}}{\pi} \frac{1}{v_{\mathrm{PQ}}} \approx g_{a \gamma \gamma} .
$$

Instead of a $\mathbf{Z}_{2}$ orbifold compactification, one could have equally considered the compactification on a $\delta$-dimensional torus [5], leading to modified coupling constants by factors of order unity. For the sake of simplicity we will always assume that the KK axion modes couple to photons with the usual PQ coupling $g_{a \gamma \gamma}$; it is trivial to insert modeldependent factors in the final result.

A few comments are now in order in connection with the effective KK Lagrangian (2.9). First, we should remark that 
the higher-dimensional PQ-breaking scale $\bar{v}_{\mathrm{PQ}}$ may be very low at the $\mathrm{TeV}$ scale, when compared to the usual fourdimensional one $v_{\mathrm{PQ}}$, i.e.,

$$
\bar{v}_{\mathrm{PQ}} \approx\left(\frac{M_{\mathrm{F}}}{M_{\mathrm{P}}}\right)^{\delta / n} v_{\mathrm{PQ}} .
$$

The suppression mechanism is very analogous to the case in which the fundamental scale of quantum gravity can be reduced to the electroweak scale in the presence of large extra dimensions [5] [cf. Eq. (1.1)]. In Eq. (2.11), the simplest setting is to consider that both gravity and axions live within the same higher-dimensional space, i.e., $\delta=n$. Second, one notices that the lowest KK state constitutes the PQ axion of the theory which determines the size of the coupling of the KK axions to photons. Finally, the KK-axion masses are given by

$$
m_{a_{0}}=m_{\mathrm{PQ}} \ll \frac{1}{R}, \quad m_{a_{\mathbf{n}}} \approx \frac{n}{R},
$$

with $n=|\mathbf{n}|=\sqrt{n_{1}^{2}+\cdots+n_{\delta}^{2}}>0$. It is interesting to observe that for the higher-dimensional scenarios under discussion, the mass spacing of the KK axions is always larger than PQ masses lying in the phenomenologically favored region, with $m_{\mathrm{PQ}} \lesssim 0.01 \mathrm{eV}$. For example, for $\delta=2$ and $M_{\mathrm{F}} \approx 100 \mathrm{TeV}$ [7], one obtains $1 / R \sim 1 \mathrm{eV}$, while for $\delta=3$ and $M_{\mathrm{F}} \approx 1 \mathrm{TeV}$, the inverse of the compactification radius reaches a much higher value, i.e., $1 / R \sim 10 \mathrm{eV}$.

The lifetime of an individual axionic KK state $a_{\mathbf{n}}$ may easily be computed from Eq. (2.6). In this way, we find

$$
\tau\left(a_{\mathbf{n}} \rightarrow \gamma \gamma\right) \approx\left(\frac{m_{\mathrm{PQ}}}{m_{a_{\mathbf{n}}}}\right)^{3} \tau\left(a_{0} \rightarrow \gamma \gamma\right) .
$$

We observe that the lifetime of the KK axion $a_{\mathbf{n}}$ decreases rapidly with the third power of its mass. For example, the lifetime of one single (solar) KK-axion mode with $m_{a_{\mathrm{n}}}$ $=10 \mathrm{keV}$ and $g_{a \gamma \gamma}=10^{-11} \mathrm{GeV}^{-1}$ (corresponding to $m_{\mathrm{PQ}}$ $\left.=10^{-1} \mathrm{eV}\right)$ is $\tau\left(a_{\mathbf{n}} \rightarrow \gamma \gamma\right) \approx 10^{12}$ days, which is 15 orders of magnitude smaller than the respective one obtained in the usual four-dimensional theories of PQ axions.

\section{SOLAR FLUX OF KALUZA-KLEIN AXIONS}

\section{A. Primakoff process}

In order to calculate the solar flux of KK axion modes we restrict ourselves to hadronic axion models where these particles do not couple to electrons at tree level. The dominant production processes will thus involve the axion-photon interaction; the axion-nucleon coupling will not be important in the Sun. The usual PQ axions are primarily produced by the Primakoff process $\gamma+Z e \rightarrow Z e+a$ where a thermal photon in the solar interior converts into an axion in the Coulomb fields of nuclei and electrons in the solar plasma. In addition, the KK modes can be produced by the photon coalescence process $\gamma \gamma \rightarrow a$. For PQ axions, this process is suppressed by the small mass and actually is kinematically for- bidden in the solar plasma because the effective photon mass (plasma frequency) is about $0.3 \mathrm{keV}$. However, with a temperature in the Sun of around $1.3 \mathrm{keV}$, the solar KK axions will be produced with masses up to several $\mathrm{keV}$, rendering the coalescence process an important contribution.

Beginning with the Primakoff process, the production cross section on a target with charge $Z e$ in a nonrelativistic plasma is found to be [17]

$$
\frac{d \sigma_{\gamma \rightarrow a}}{d \Omega}=\frac{g_{a \gamma \gamma}^{2} Z^{2} \alpha}{8 \pi} \frac{|\mathbf{k} \times \mathbf{p}|^{2}}{\mathbf{q}^{4}} \frac{\mathbf{q}^{2}}{\mathbf{q}^{2}+\kappa^{2}},
$$

where $\mathbf{k}$ is the photon momentum, $\mathbf{p}$ the axion momentum, and $\mathbf{q}=\mathbf{k}-\mathbf{p}$ the momentum transfer. The last factor takes account of screening effects where the Debye-Hückel screening scale is given by

$$
\kappa^{2}=\frac{4 \pi \alpha}{T} \frac{\rho}{m_{u}}\left(Y_{e}+\sum_{j} Z_{j}^{2} Y_{j}\right) .
$$

In Eq. (3.2), $\rho$ is the mass density, $m_{u}$ the atomic mass unit (approximately the proton mass), $Y_{e}$ the number of electrons per baryon in the medium, and $Y_{j}$ the number of various nuclear species $j$ per baryon with nuclear charge $Z_{j}$. The medium is assumed to be nonrelativistic, and recoil effects by the targets have been neglected since typical photon energies of a few $\mathrm{keV}$ are much smaller than even the electron mass. It turns out that we have the approximate relation, $\kappa$ $\approx 7 T$, between the screening scale $\kappa$ and the temperature $T$ in the relevant regions of the Sun.

Summing over all target species of the medium, the photon-axion transition rate is finally

$$
\Gamma_{\gamma \rightarrow a}=\frac{g_{a \gamma \gamma}^{2} T \kappa^{2}}{32 \pi^{2}} \frac{|\mathbf{k}|}{\omega} \int d \Omega \frac{|\mathbf{k} \times \mathbf{p}|^{2}}{\mathbf{q}^{2}\left(\mathbf{q}^{2}+\kappa^{2}\right)},
$$

where $\omega$ is the photon energy and the factor $|\mathbf{k}| / \omega$ is the relative velocity between photons and target particles. The angular integration can be performed explicitly, leading to

$$
\begin{aligned}
\Gamma_{\gamma \rightarrow a}= & \frac{g_{a \gamma \gamma}^{2} T \kappa^{2}}{32 \pi} \frac{k}{\omega}\left\{\frac{\left[(k+p)^{2}+\kappa^{2}\right]\left[(k-p)^{2}+\kappa^{2}\right]}{4 k p \kappa^{2}}\right. \\
& \left.\times \ln \left[\frac{(k+p)^{2}+\kappa^{2}}{(k-p)^{2}+\kappa^{2}}\right]-\frac{\left(k^{2}-p^{2}\right)^{2}}{4 k p \kappa^{2}} \ln \left[\frac{(k+p)^{2}}{(k-p)^{2}}\right]-1\right\},
\end{aligned}
$$

where $k=|\mathbf{k}|$ and $p=|\mathbf{p}|$.

The effective "photon mass" in the medium, the plasma frequency, is small in the Sun, typically about $0.3 \mathrm{keV}$, while the temperature near the solar center is $T=1.3 \mathrm{keV}$ and typical photon energies are $3 T \approx 4 \mathrm{keV}$. Therefore, we ignore the plasma frequency and treat photons as strictly massless. In a photon-axion transition the energy is conserved because we ignore recoil effects. Therefore, we use $k=E$ with $E$ the axion energy and $p=\sqrt{E^{2}-m^{2}}$ so that finally 


$$
\begin{aligned}
\Gamma_{\gamma \rightarrow a}= & \frac{g_{a \gamma \gamma}^{2} T \kappa^{2}}{32 \pi}\left\{\frac{\left(m^{2}-\kappa^{2}\right)^{2}+4 E^{2} \kappa^{2}}{4 E p \kappa^{2}} \ln \left[\frac{(E+p)^{2}+\kappa^{2}}{(E-p)^{2}+\kappa^{2}}\right]\right. \\
& \left.-\frac{m^{4}}{4 E p \kappa^{2}} \ln \left[\frac{(E+p)^{2}}{(E-p)^{2}}\right]-1\right\} .
\end{aligned}
$$

Note that the expression in curly brackets expands for small momenta as

$$
\{\cdots\}=\frac{8 p^{2}}{3\left(\kappa^{2}+m^{2}\right)}+\mathcal{O}\left(p^{4}\right)
$$

so that the emission of slow-moving axions is suppressed.

The axion flux at Earth, differential with regard to the axion energy $E$, is then found by multiplying the transition rate with the blackbody photon flux in the Sun, and integrating over a standard solar model,

$$
\Phi_{a}=\frac{d F_{a}}{d E}=\frac{1}{4 \pi d_{\odot}^{2}} \int_{\text {sun }} d^{3} \mathbf{r} \Gamma_{\gamma \rightarrow a} \frac{1}{\pi^{2}} \frac{E^{2}}{e^{E / T}-1} .
$$

Here $T$ and $\kappa^{2}$ depend on the location in the Sun and $d_{\odot}$ $=1.50 \times 10^{13} \mathrm{~cm}$ is the distance to the Sun. We stress that no velocity factor appears for massive axions because in a stationary situation all axions produced per second must traverse a spherical shell around the Sun within one second.

In Ref. [18] an approximation formula for the axion flux at Earth was given which we slightly modify and extend to the case of massive $\mathrm{KK}$ axions

$$
\begin{aligned}
\Phi_{a}= & 4.20 \times 10^{10} \mathrm{~cm}^{-2} \mathrm{~s}^{-1} \mathrm{keV}^{-1}\left(\frac{g_{a \gamma \gamma}}{10^{-10} \mathrm{GeV}^{-1}}\right)^{2} \\
& \times \frac{E p^{2}}{e^{E / 1.1}-0.7}(1+0.02 \mathrm{~m})
\end{aligned}
$$

where $E, p$ and $m$ are to be measured in $\mathrm{keV}$. This approximation formula is typically good to better than $\pm 15 \%$ for all relevant conditions, and even better than a few percent for the most relevant case of axion masses of larger than a few keV. In Fig. 1 we show the energy dependence of the flux of massive KK axions at Earth for three typical choices of the axion mass: $m=5,10$ and $15 \mathrm{keV}$.

\section{B. Photon coalescence}

In order to calculate the production rate of axions from the process $\gamma \gamma \rightarrow a$ in a thermal medium, we approximate the Bose-Einstein photon distribution by a Maxwell-Boltzmann one, i.e., we use $e^{-\omega / T}$ instead of $1 /\left(e^{\omega / T}-1\right)$ for the photon occupation number. This approximation is justified since we are interested only in axion masses and thus axion energies of order the temperature or larger. The production rate of axions of energy $E$ per unit volume and unit energy interval is then found to be

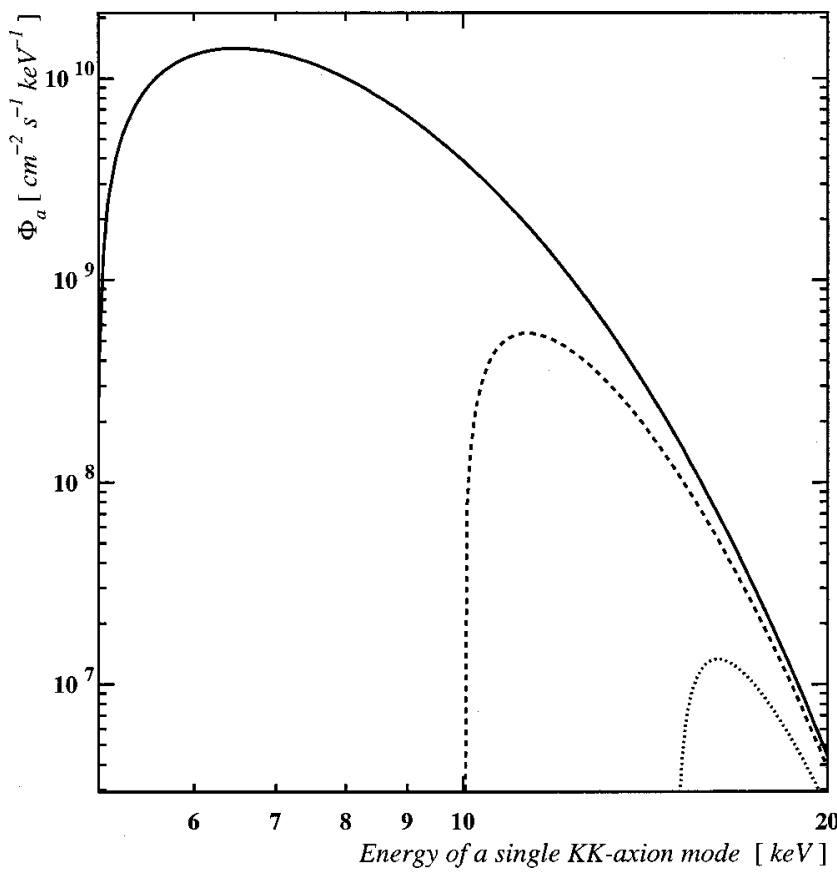

FIG. 1. Energy dependence of the solar flux of KK axions at a distance of $1 \mathrm{AU}$ due to Primakoff process, assuming a KK axion mass $m=5$ (solid line), 10 (dashed line), and 15 (dotted line) $\mathrm{keV}$, and $g_{a \gamma \gamma}=10^{-10} \mathrm{GeV}^{-1}$.

$$
\frac{d N_{a}}{d E}=\frac{g_{a \gamma \gamma}^{2} m^{4}}{128 \pi^{3}} p e^{-E / T}
$$

where again $p=\sqrt{E^{2}-m^{2}}$ is the axion momentum. Integrating this expression over a standard solar model we find the axion flux at Earth. It is approximately represented by

$$
\begin{aligned}
\Phi_{a}= & 1.68 \times 10^{9} \mathrm{~cm}^{-2} \mathrm{~s}^{-1} \mathrm{keV}^{-1}\left(\frac{g_{a \gamma \gamma}}{10^{-10} \mathrm{GeV}^{-1}}\right)^{2} m^{4} p \\
& \times\left(\frac{10}{0.2+E^{2}}+1+0.0006 E^{3}\right) e^{-E},
\end{aligned}
$$

where again $m, E$ and $p$ are to be taken in $\mathrm{keV}$. For $1 \mathrm{keV}$ $<E<16 \mathrm{keV}$ the quality of the approximation is better than $5 \%$. Both lower and higher energies are irrelevant for our purposes. In Fig. 2 we display numerical estimates of the flux of KK axions at Earth as a function of their energy for three selected values of axion mass: $m=5,10$ and $15 \mathrm{keV}$. A direct comparison of Fig. 1 with Fig. 2 reveals that the photon coalescence process becomes more important than the Primakoff one for the heavier KK-axion modes.

\section{Axion limit from solar energy loss}

As a next step we consider the energy loss of the Sun as a function of $g_{a \gamma \gamma}$. To this end we first calculate the solar axion luminosity as a function of the KK axion mass

$$
L_{a}(m)=4 \pi d_{\odot}^{2} \int_{m}^{\infty} d E E \Phi_{a}(E)
$$




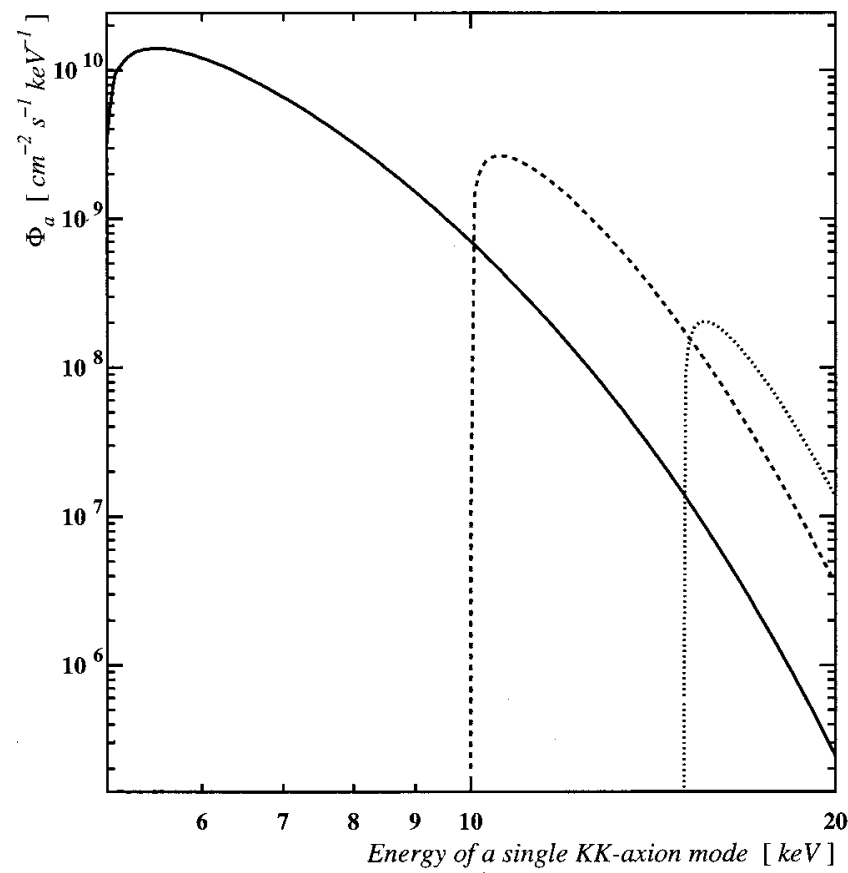

FIG. 2. Energy dependence of the solar flux of KK axions at a distance of $1 \mathrm{AU}$ due to photon coalescence, assuming a KK axion mass $m=5$ (solid line), 10 (dashed line), and 15 (dotted line) keV, and $g_{a \gamma \gamma}=10^{-10} \mathrm{GeV}^{-1}$.

for the two processes. Then we need to sum over all KK modes with their different masses. Instead, we integrate over the density of modes which is $R^{\delta}$, where $R$ is the compactification radius and $\delta$ the number of compactified dimensions. Therefore, the axion luminosity is

$$
L_{a}=\frac{2 \pi^{\delta / 2}}{\Gamma(\delta / 2)} R^{\delta} \int_{0}^{\infty} d m m^{\delta-1} L_{a}(m),
$$

where the first factor is the surface of the $\delta$ dimensional unit sphere, i.e., 2 for $\delta=1,2 \pi$ for $\delta=2$ and $4 \pi$ for $\delta=3$. Numerically, we write the result in the form

$$
L_{a}=A L_{\odot}\left(\frac{g_{a \gamma \gamma}}{10^{-10} \mathrm{GeV}^{-1}}\right)^{2}\left(\frac{R}{\mathrm{keV}^{-1}}\right)^{\delta},
$$

where $L_{\odot}$ is the luminosity of the Sun and the values of the coefficients $A$ for the two processes and different dimensions $\delta$ are given in Table I. It depends on $\delta$ which of the processes is more important.

Helioseismology implies that a novel energy-loss mechanism of the Sun must not exceed something like $0.2 L_{\odot}$ [19]. This limit translates into the constraint

TABLE I. Coefficients $A$ for Eq. (3.13).

\begin{tabular}{lccc}
\hline \hline & Primakoff & Coalescence & Sum \\
\hline$\delta=1$ & 0.015 & 0.0033 & 0.018 \\
$\delta=2$ & 0.12 & 0.067 & 0.19 \\
$\delta=3$ & 0.99 & 1.06 & 2.1 \\
\hline \hline
\end{tabular}

$$
\left(\frac{g_{a \gamma \gamma}}{10^{-10} \mathrm{GeV}^{-1}}\right)\left(\frac{R}{\mathrm{keV}^{-1}}\right)^{\delta / 2}<\left\{\begin{array}{lll}
3.3 & \text { for } & \delta=1, \\
1.0 & \text { for } & \delta=2, \\
0.31 & \text { for } & \delta=3 .
\end{array}\right\}
$$

As an example we use the simplest setting of $\delta=n=2$ large extra dimensions, with $M_{\mathrm{F}}=100 \mathrm{TeV}$ and $R=10^{3} \mathrm{keV}^{-1}$, leading to $g_{a \gamma \gamma}<10^{-13} \mathrm{GeV}^{-1}$. For $\delta=n=3$ large extra dimensions, with $M_{\mathrm{F}}=1 \mathrm{TeV}$ and $R=10^{2} \mathrm{keV}^{-1}$, we get an even better limit of $g_{a \gamma \gamma}<0.3 \times 10^{-13} \mathrm{GeV}^{-1}$. This is to be compared with the solar PQ axion limit of $g_{a \gamma \gamma}$ $<10^{-9} \mathrm{GeV}^{-1}$ [20]. Of course, the KK limits could have been estimated by simply scaling the standard limit with the multiplicity of KK modes and observing that the maximum allowed mass is a few keV before the solar flux gets suppressed by the kinematic threshold.

\section{FLUX OF DECAY PHOTONS}

\section{A. Numerical estimates}

The KK axions emerging from the Sun are neither nonrelativistic nor strongly relativistic. The average speed is 0.95 (in units of the speed of light) for $m=1 \mathrm{keV}, 0.79$ for 3 $\mathrm{keV}, 0.66$ for $5 \mathrm{keV}, 0.57$ for $7 \mathrm{keV}$, and 0.51 for $9 \mathrm{keV}$. Therefore, the decay photons will have a considerable angular spread relative to the direction of the Sun. The event rate in a detector thus depends crucially on its geometry. For our simple estimate we will assume that the detector consists of a volume $V$, and that any $\mathrm{x}$-ray produced within this volume will be detected with unit efficiency, independently of its direction.

In view of the solar energy-loss limits derived above we further note that even $\mathrm{keV}$-mass axions are long-lived relative to the Sun-Earth distance so that the axion flux on its way to Earth is not significantly diminished by radiative decays. Therefore, at any given time the total number of solar axions of mass $m$ per unit energy interval in the detector is

$$
\frac{d N_{a}}{d E}=\frac{V \Phi_{a}}{v},
$$

where $v=p / E$ is the axion velocity. In the laboratory frame they decay with a rate $(m / E) \Gamma_{a \rightarrow \gamma \gamma}=\left(g_{a \gamma \gamma}^{2} / 64 \pi\right) m^{4} / E$, each decay producing 2 photons with energies which are uniformly distributed in the range $(E-p) / 2 \leqslant \omega \leqslant(E+p) / 2$. This implies that in order to get a decay photon of energy $\omega$ the parent axion must have $E \geqslant \omega+m^{2} / 4 \omega$ and that the photon energies from a given axion decay are spread over an interval of length $p$. Altogether, then, we find, for the differential event rate of decay photons from axions of mass $m$,

$$
\frac{d N_{\gamma}(m, \omega)}{d \omega}=\Gamma_{a \rightarrow \gamma \gamma} m V \int_{\omega+m^{2} / 4 \omega}^{\infty} d E \frac{2 \Phi_{a}}{p^{2}} .
$$

Finally, in order to obtain the total event rate due to all modes of the tower of KK modes we proceed as before by integrating over the density of modes so that 
TABLE II. Coefficients for the rate Eq. (4.4) and for the spectra of Eq. (4.5).

\begin{tabular}{lcccc}
\hline \hline & $A_{\delta}\left[\mathrm{day}^{-1}\right]$ & $a\left[\mathrm{keV}^{-1}\right]$ & $b$ & $\langle\omega\rangle[\mathrm{keV}]$ \\
\hline$\delta=1$ & 0.16 & 0.0338 & 3.8 & 5.3 \\
$\delta=2$ & 4.7 & 0.0107 & 4.5 & 6.1 \\
$\delta=3$ & 100.0 & 0.0037 & 5.1 & 6.8 \\
\hline \hline
\end{tabular}

$$
\frac{d N_{\gamma}(\omega)}{d \omega}=\frac{2 \pi^{\delta / 2}}{\Gamma(\delta / 2)} R^{\delta} V \int_{0}^{\infty} d m m^{\delta} \Gamma_{a \rightarrow \gamma \gamma} \int_{\omega+m^{2} / 4 \omega}^{\infty} d E \frac{2 \Phi_{a}}{p^{2}} .
$$

Numerically, we write this in the form

$$
\frac{d N_{\gamma}(\omega)}{d \omega}=A_{\delta}\left(\frac{g_{a \gamma \gamma}}{10^{-10} \mathrm{GeV}^{-1}}\right)^{4}\left(\frac{R}{\mathrm{keV}^{-1}}\right)^{\delta}\left(\frac{V}{\mathrm{~m}^{3}}\right) f_{\delta}(\omega),
$$

where $A_{\delta}$ is a rate given in Table II and $f_{\delta}(\omega)$ is a spectrum with its integral normalized to unity. These normalized functions are surprisingly well approximated by the simple analytic form

$$
f_{\delta}(\omega)=a \omega^{b} e^{-0.9 \omega}
$$

where $a$ and $b$ are given in Table II for each $\delta$. Of course, $\omega$ is understood in $\mathrm{keV}$. In Table II we also give the average photon energies. In particular, as can be seen from Fig. 3, the energy distributions of the decay photons are shifted towards the few keV energy range.

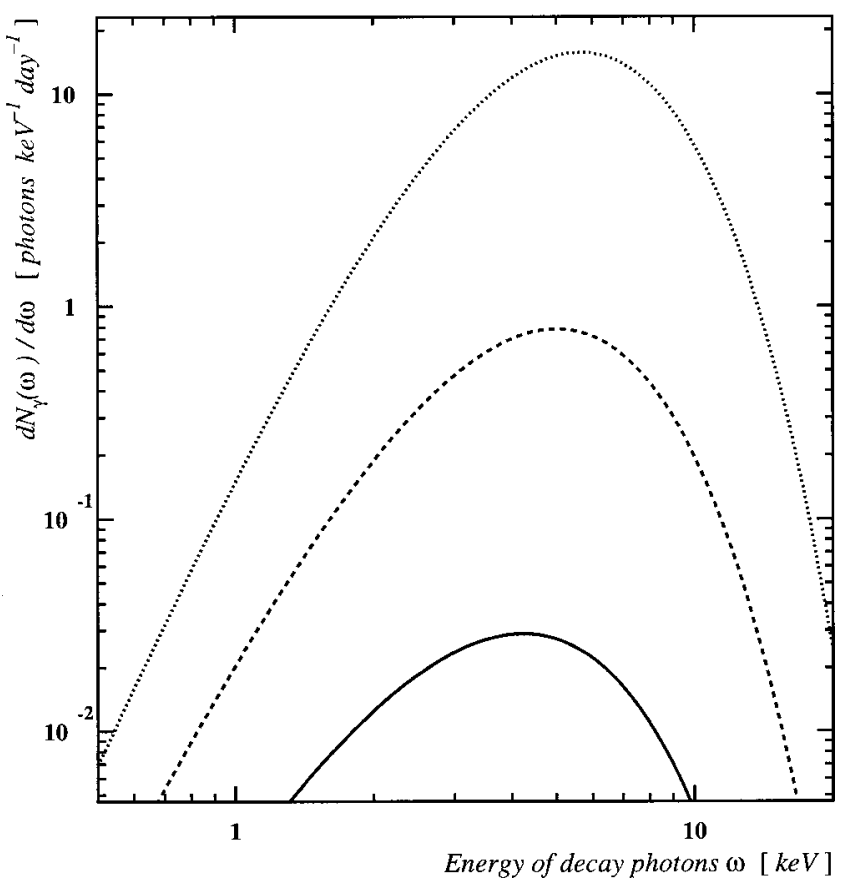

FIG. 3. Energy dependence of $d N_{\gamma}(\omega) / d \omega$, for $\delta=1$ (solid line), $\delta=2$ (dashed line), and $\delta=3$ (dotted line), with $g_{a \gamma \gamma}$ $=10^{-10} \mathrm{GeV}^{-1}, R=1 \mathrm{keV}^{-1}$, and $V=1 \mathrm{~m}^{3}$.

\section{B. Experimental sensitivity}

On the experimental side, we assume a $1 \mathrm{~m}^{3}$ detector of the Micromegas type. This is a new kind of gas detector which can be used to measure photon interactions with good space and energy resolution [21]. A small detector of this kind, with a surface of $15 \times 15 \mathrm{~cm}^{2}$, was used in Saclay (on the surface) and measured 1.2 neutral particles per second in a $1 \mathrm{keV}$ wide energy interval centered at $1 \mathrm{keV}$. At these energies, practically all photons entering the chamber interact in the gas, so we have a measurement of the neutral particle flux through a surface of $15 \times 15 \mathrm{~cm}^{2}$, which is about 53 neutral particles $/ \mathrm{m}^{2} / \mathrm{sec}$ [22].

In the search for axion decays into two gammas, the background originates from two neutral particles interacting in the gas within the resolving time of the chamber. Therefore, one can choose the gas so that the mean absorption length of $1 \mathrm{keV}$ photons is $0.3 \mathrm{~cm}$ [23]. As a result, the interaction points of the two photons from axion decay will be very close to each other, in a cell with volume $\Delta x \Delta y \Delta z$ $=1 \mathrm{~cm}^{3}$. In the Micromegas chamber $\Delta x$ and $\Delta y$ are measured directly and $\Delta z$ is measured from the time interval between the two signals. For $\Delta z=1 \mathrm{~cm}$, the time interval is $2 \times 10^{-7} \mathrm{sec}$. Thus, in a small cell of $1 \mathrm{~cm}^{3}$ volume, the rate of events from two uncorrelated neutral particles is 5.6 $\times 10^{-12}$ events/sec. As there are $10^{6}$ cells when going from $1 \mathrm{~cm}^{3}$ cell size to $1 \mathrm{~m}^{3}$ size of the detector, the background rate becomes 0.5 event per day.

At this point, we should remark that we have not used two additional criteria to reduce the background:

(a) Real photons in the $\mathrm{keV}$ region entering the detector from outside will interact very close to the detector walls. If one requires that the events occur in a fiducial volume at some distance (few $\mathrm{cm}$ ) from the walls, only photons generated inside the detector volume are important.

(b) If axions are non-relativistic, the two photons will have approximately equal energies.

Of course, a more precise estimate of the background requires a measurement with a realistic detector in the environment where the experiment is going to be performed.

Applying now Eq. (4.4) to the simplest setting of $\delta=n$ $=2$ large extra dimensions, with $M_{\mathrm{F}}=100 \mathrm{TeV}$ and $R$ $=10^{3} \mathrm{keV}^{-1}$, we find the rate

$$
R_{\gamma} \approx 0.05 \text { event day }{ }^{-1} \mathrm{~m}^{-3}\left(\frac{g_{a \gamma \gamma}}{10^{-12} \mathrm{GeV}^{-1}}\right)^{4}
$$

Consequently, the suggested terrestrial detector outlined above will be sensitive to an effective $a \gamma \gamma$ coupling $g_{a \gamma \gamma}$ $\lesssim 2.0 \times 10^{-12} \mathrm{GeV}^{-1}$, corresponding to a fundamental PQ mass $m_{\mathrm{PQ}} \approx 10^{-2} \mathrm{eV}$. In particular, for $\delta=n=3$ large extra dimensions, with $M_{\mathrm{F}}=1 \mathrm{TeV}$ and $R=10^{2} \mathrm{keV}^{-1}$, we obtain an estimate for the rate

$$
R_{\gamma} \approx 1.0 \text { event day }{ }^{-1} \mathrm{~m}^{-3}\left(\frac{g_{a \gamma \gamma}}{10^{-12} \mathrm{GeV}^{-1}}\right)^{4}
$$

From this last result, one can readily see that the axion detector will be maximally sensitive to an effective $a \gamma \gamma$ cou- 
pling $g_{a \gamma \gamma} \leq 6.0 \times 10^{-13} \mathrm{GeV}^{-1}$, corresponding to a fundamental PQ mass $m_{\mathrm{PQ}} \approx 3.0 \times 10^{-3} \mathrm{eV}$.

Finally, it would be interesting to know whether measurements of $\gamma$-rays coming from the Sun could impose severe constraints on the $2 \gamma$-decay mode of axions and hence on the parameters of the higher-dimensional axionic models under consideration [20]. According to recent analyses [24], the solar x-ray luminosity in the range of interest to us, i.e., above $0.4 \mathrm{keV}$, is

$$
L_{\mathrm{x} \text {-rays }} \approx 10^{9} \mathrm{event} / \mathrm{cm}^{2} / \mathrm{sec} \approx 10^{17} \mathrm{events} / \mathrm{day} / \mathrm{m}^{2} .
$$

As the decay path available for solar axions is the distance to the Sun of $1.5 \times 10^{11} \mathrm{~m}$, the $\mathrm{x}$-ray luminosity is by many orders of magnitude larger than the one expected from the decays of the KK axions.

\section{Laboratory limits on solar axions}

Lowest level underground experiments [25] searching for weakly interacting massive particles and other particles offer independent limits on the effective axion-to-photon coupling $g_{a \gamma \gamma}$. Specifically, these experiments report the following lower limit on the integrated event rate in the energy range below $10 \mathrm{keV}$ :

$$
R_{\gamma}^{\exp } \lesssim 20000 \text { event day }{ }^{-1} \mathrm{~m}^{-3} .
$$

These highly sensitive experiments measure the deposited energy but they are unable to distinguish between 1-prong and 2-prong events.

Applying Eq. (4.9) to Eqs. (4.6) and (4.7), we are able to derive for the first time experimental limits on $g_{a \gamma \gamma}$ in theories with $\mathrm{KK}$ axions. In this way, we find the upper limits

$$
g_{a \gamma \gamma} \lesssim 2.5 \times 10^{-11} \mathrm{GeV}^{-1},
$$

for $\delta=2$ and $M_{F}=100 \mathrm{TeV}$, and

$$
g_{a \gamma \gamma} \lesssim 1.2 \times 10^{-11} \mathrm{GeV}^{-1}
$$

for $\delta=3$ and $M_{F}=1 \mathrm{TeV}$. Evidently, our suggested underground detector will improve at least by one order of magnitude the present experimental limits which we derived in Eqs. (4.10) and (4.11). The latter upper limits should also be contrasted with the weaker upper bound, $g_{a \gamma \gamma} \lesssim 6.0$ $\times 10^{-10} \mathrm{GeV}^{-1}$, which is obtained from recent experimental searches for conventional PQ axions coming from the Sun [26].

\section{CONCLUSIONS}

We have examined the potential of an underground detector shielded from cosmic-ray backgrounds for detecting $\mathrm{KK}$ axions coming from the Sun. The solar KK axions may be produced via the Primakoff process $\gamma+Z e \rightarrow Z e+a$ or via the photon coalescence process $\gamma \gamma \rightarrow a$. In either case, we have calculated the expected flux of the KK axions, as well as estimated possible limits derived from helioseismology. We find that solar KK axions might lead to observable signatures in terrestrial experiments. In fact, the characteristic $2 \gamma$-decay mode of the KK axions offers a unique possibility to drastically reduce the background by coincidental triggering both of the emitted photons. Our elaborate estimates have shown that a terrestrial detector of $1 \mathrm{~m}^{3}$ size may be sensitive to a fundamental PQ-axion mass up to $10^{-2} \mathrm{eV}$, which amounts to having an effective axion-photon coupling $g_{a \gamma \gamma} \approx 2.0 \times 10^{-12} \mathrm{GeV}^{-1}$, in theories with 2 large extra dimensions and a fundamental quantum-gravity scale $M_{\mathrm{F}}$ $=100 \mathrm{TeV}$. In particular, in theories with 3 large compact dimensions with $M_{\mathrm{F}}=1 \mathrm{TeV}$, the suggested detector is capable of probing $\mathrm{PQ}$-axion masses up to $3.0 \times 10^{-3} \mathrm{eV}$, corresponding to an effective axion-photon coupling $g_{a \gamma \gamma} \approx 6.0$ $\times 10^{-13} \mathrm{GeV}^{-1}$. Most importantly, the experimental detector under discussion will considerably improve, at least by one order of magnitude, the corresponding experimental limits on $g_{a \gamma \gamma}$ in theories with KK axions, which we derived in Eqs. (4.10) and (4.11) based on present data obtained from underground experiments.
[1] I. Antoniadis, Phys. Lett. B 246, 377 (1990).

[2] E. Witten, Nucl. Phys. B471, 135 (1996).

[3] P. Hořava and E. Witten, Nucl. Phys. B460, 506 (1996); B475, 94 (1996).

[4] J.D. Lykken, Phys. Rev. D 54, 3693 (1996).

[5] N. Arkani-Hamed, S. Dimopoulos, and G. Dvali, Phys. Lett. B 429, 263 (1998); Phys. Rev. D 59, 086004 (1999); I. Antoniadis, N. Arkani-Hamed, S. Dimopoulos, and G. Dvali, Phys. Lett. B 436, 257 (1998).

[6] K.R. Dienes, E. Dudas, and T. Gherghetta, Phys. Lett. B 436, 55 (1998); Nucl. Phys. B537, 47 (1999).

[7] L.J. Hall and D. Smith, Phys. Rev. D 60, 085008 (1999).

[8] N. Arkani-Hamed, S. Dimopoulos, G. Dvali, and J. MarchRussell, hep-ph/9811448.

[9] K.R. Dienes, E. Dudas, and T. Gherghetta, Nucl. Phys. B557, 25 (1999).

[10] A. Pilaftsis, Phys. Rev. D 60, 105023 (1999); G. Dvali and
A.Yu. Smirnov, Nucl. Phys. B563, 63 (1999); A. Faraggi and M.E. Pospelov, Phys. Lett. B 458, 237 (1999); A. Ioannisian and A. Pilaftsis, Phys. Rev. D 62, 066001 (2000); Y. Grossman and M. Neubert, Phys. Lett. B 474, 361 (2000); A. Ioannisian and J.W.F. Valle, hep-ph/9911349; E. Ma, M. Raidal, and U. Sarkar, Phys. Rev. Lett. (to be published), hep-ph/ 0006046; R.N. Mohapatra and A. Pérez-Lorenzana, hep-ph/0006278.

[11] S. Chang, S. Tazawa, and M. Yamaguchi, Phys. Rev. D 61, 084005 (2000).

[12] K.R. Dienes, E. Dudas, and T. Gherghetta, Phys. Rev. D 62, 105023 (2000).

[13] C.E. Aalseth et al., CERN-SPSC 99-21/P312 (9.8.1999); K. Zioutas et al., Nucl. Instrum. Methods Phys. Res. A 425, 480 (1999), and references therein.

[14] A.P. Zhitnitskii, Sov. J. Nucl. Phys. 31, 260 (1980); M. Dine, W. Fischler, and M. Srednicki, Phys. Lett. 104B, 199 (1981). 
[15] J.E. Kim, Phys. Rev. Lett. 43, 103 (1979); M. Shifman, A. Vainshtein, and V. Zakharov, Nucl. Phys. B166, 493 (1980).

[16] G. Raffelt, Annu. Rev. Nucl. Part. Sci. 49, 163 (1999).

[17] G.G. Raffelt, Phys. Rev. D 33, 897 (1986).

[18] K. van Bibber et al., Phys. Rev. D 39, 2089 (1989).

[19] H. Schlattl, A. Weiss, and G. Raffelt, Astropart. Phys. 10, 353 (1999).

[20] G. Raffelt, Stars as Laboratories for Fundamental Physics (University of Chicago Press, Chicago, 1996).

[21] Y. Giomataris, P. Rebourgeard, J.P. Robert, and G. Charpak, Nucl. Instrum. Methods Phys. Res. A 376, 29 (1996).

[22] Measured in Saclay with the Group of Y. Giomataris.
[23] See Particle Data Group, C. Caso et al., Eur. Phys. J. C 3, 1 (1998), p. 152, Fig. 24.1.

[24] P. Foukal, Solar Astrophysics (Wiley, New York, 1990), p. 435.

[25] A. Morales, Nucl. Phys. B (Proc. Suppl.) 87, 477 (2000); L. Baudis, A. Dietz, B. Majorovits, F. Schwamm, H. Strecker, and H.V. Klapdor-Kleingrothaus, Phys. Rev. D (to be published), astro-ph/0008339.

[26] S. Moriyama, M. Minowa, T. Namba, Y. Inoue, Y. Takasu, and A. Yamamoto, Phys. Lett. B 434, 147 (1998); The SOLAX Collaboration, F.T. Avignone III et al., Phys. Rev. Lett. 81, 5068 (1998). 